\title{
Measurement Validation of Fuzzy-like Propagation Models for Wireless Cellular Networks in Metropolitan Environment
}

\author{
O.O. Shoewu ${ }^{1 *}$, M.A. Adedoyin ${ }^{1}$, L.A. Akinyemi ${ }^{1,2}$, \\ L.I. Oborkhale ${ }^{3}$ and E.U. Udo ${ }^{3}$ \\ ${ }^{1}$ Department of Electronic and Computer Engineering, Lagos State University, Nigeria \\ ${ }^{2}$ Department of Electrical Engineering, University of Cape Town, South Africa \\ ${ }^{3}$ Department of Electrical and Electronics Engineering, Michael Okpara University of Agriculture, Nigeria \\ Email: oluwagbemiga.shoewu@ @asu.edu.ng ( Oluwagbemiga O. Shoewu) \\ *Correspondence: oluwagbemiga.shoewu@ lasu.edu.ng
}

Received: $17^{\text {th }}$ July 2017

Accepted: $13^{\text {th }}$ December 2017

Published: $16^{\text {th }}$ March 2018

https://doi.org/10.47545/etrj.2018.3.1.037

\begin{abstract}
Increasing demand for radio channels has brought about generational developments which emanated from the explosive requirement for wireless services in a metropolitan environment is one of the major challenges facing the path loss propagation prediction. However, this prediction is necessary for link budget and in the determination of the coverage area of a particular base station. Therefore, in this paper, a fuzzy-logic based path loss prediction model is proposed for accurate link budget and optimal coverage determination. Also, a drive test is conducted and data were collected for non urban, urban, suburban, ex-urban, dense-urban, microurban and peri urban areas in Lagos metropolitan environment for training the proposed model. Network parameters were varied during the performance evaluation process for the $900 \mathrm{MHz}$ and $1800 \mathrm{MHz}$ bands. Results show that the mean path loss for different environments is higher for the $900 \mathrm{MHz}$ band compared with $1800 \mathrm{MHz}$ band and this shows that propagation in the $900 \mathrm{MHz}$ environment is characterized with more challenges and dynamic propagation conditions than in 1800MHz. Additionally, the urban environment gives the highest value for the constant offset being $60 \mathrm{~dB}$ for $900 \mathrm{MHz}$ but the ex-urban environment has a constant offset of $64 \mathrm{~dB}$ in $1800 \mathrm{MHz}$.
\end{abstract}

Keywords: Drive-Test, Fuzzy-Logic, Metropolitan, Path loss,

\section{INTRODUCTION}

The rapid growth in wireless telecommunication services results in increased desire for next generation services by mobile subscribers. However, this event has resulted in the need for proper network coverage predictions ${ }^{[1]}$. It has been shown in the literature that propagation prediction models such as Okumura-Hata and the WalfischIkegami (WI) are based on substantial experimental data collection and statistical analysis, which help in computing the receive signal level in a particular propagation medium ${ }^{[2]}$. Also, mobile communication requires high-quality received signal at the receivers, however, some receiving points provide weak signal due to obstructions such as buildings and trees between a transmitter and a receiver ${ }^{[3]}$. In the design of wireless communication systems, the knowledge of the propagation characteristics of a wireless radio channel is very vital in a given environment ${ }^{[4]}$. Moreover, path loss information is very important for link budget and in order to determine the coverage of a particular base station. Path loss is the attenuation that a signal suffers as it journeys from the transmitter to the receiver. Generally, some parameter estimations can only be obtained by field measurements if all the necessary pieces of information such as propagation predictions are not available, which in most cases involve drive test and is time consuming apart from being expensive.

Basically, path loss prediction models (PLPMs) are divided into empirical, site-specific propagation and theoretical models ${ }^{[4]}$. Empirical models such as Hata, Okumura and Egli models are set of algebraic equations derived from extensive field measurements. These models are accurate for environments with similar characteristics as those where the measurements were made. The input parameters for the empirical models are usually qualitative and not very specific such as a dense urban terrain, a rural terrain and some ideal environments. This model has a challenge in that it can never be used for different environments without an 
adequate modification. Site-specific propagation models are derived from numerical methods such as raytracing method (RTM) and the finite difference time-domain (FDTD) method. The input parameters are very detailed and accurate. However, this method has a very high disadvantage because it has large computational overheads. Theoretical models such as free space and plane earth propagation models are derived based on the physical laws of wave propagation. They are more efficient than the site-specific models and the empirical models.

\subsection{Literature Review}

The authors in ${ }^{[4]}$, present a comparative analysis of path loss prediction models for urban macro-cellular environments. Three path loss prediction models were used in the prediction of path loss, which are: free space, Hata and Egli. The calculated path loss values were compared with practical measured data obtained from a Visafone base station located in Uyo, Nigeria. The comparative analysis shows that the mean square errors (MSEs) for free space, Hata and Egli were $16.24 \mathrm{~dB}, 2.37 \mathrm{~dB}$ and $8.40 \mathrm{~dB}$ respectively. The presented results show that the Hata's model is the most accurate and reliable path loss prediction model for macro-cellular urban propagation environments. In ${ }^{[5]}$, the authors give the comparison between measured and predicted path loss for mobile communication in Malaysia. The path loss is measured in four different locations and results are obtained from different six models: Lee model, Log-normal shadowing, Stanford University interim (SUI) model, COST231 Hata model, the electronic communication committee (ECC)-33 and Egli model. Their measured results show that the SUI and the log-normal models give a better prediction and can be used to estimate path loss for prediction of mobile coverage in a microcell in Malaysia. The aspect of path loss prediction in wireless communication system using fuzzy logic is presented in ${ }^{[6]}$. Binary phase shift keying (BPSK) modulated signal is used to find the path loss using HATA empirical formula derived with the help of the Okumura curve for urban, suburban and rural areas. The identified values are given and Defuzzification is performed using faster and flexible centre of sum method. The inferred results show maximum loss in case of urban and increases at an average rate of $10 \mathrm{~dB}$ per decade with distance. In ${ }^{[7]}$, the authors propose a method to predict path loss for cellular mobile communication systems using fuzzy logic. The propagation medium is classified in to several well-established propagation environments defined as a fuzzy set such as free space, flat area terrain, light vegetation terrain, heavy vegetation terrain, and village terrain. A unique mean path loss exponent is assigned to each propagation environment, which is established by means of the experimental. Fuzzy logic is used to determine the unknown environment, which is obtained using linguistic rules that provide a fine tuning of the known propagation environments. The authors in ${ }^{[8]}$ propose some modifications to the COST 231 WI model to reflect the information related to building and terrain heights of practical environments. A dual slope propagation model is developed based on the modified WI model for small cells with electrical and mechanical tilt. To validate the proposed model, propagation measurements are performed in a metropolitan area using commercially deployed small cells. The authors also suggest a procedure to compute the path loss from measurement data considering transmit antenna beam patterns, and determine the break point for the proposed dual slope model using measurement results. In ${ }^{[9]}$, the authors perform comparative analysis of the basic models and artificial neural network based model for path loss prediction. Data were acquired in a drive test through selected rural and suburban routes in Minna, Nigeria and environs as dataset required for training artificial neural network (ANN) model. Multilayer perception (MLP) network parameters were varied during the performance evaluation process, and the weight and bias values of the best performed MLP network were extracted for the development of the ANN based path loss models for two different routes, namely rural and suburban routes. The performance of the developed ANN based path loss model was compared with some of the existing techniques and modified techniques. The basic empirical path loss models considered are Hata, Egli, COST-231, Ericsson models and modified path loss approach.

\subsection{Contributions and Organization}

Different from the existing works and conventional models, in this paper, we focus on path loss prediction model based on fuzzy logic in Lagos, Nigeria. The contribution of this paper is threefold with respect to existing works, as summarized below:

I. The development of a fuzzy-logic based path loss prediction model for metropolitan environments.

II. A drive test was conducted to acquire data required for training the proposed model for seven environments - non urban, urban, suburban, ex-urban, dense-urban, micro-urban and peri urban areas in Lagos metropolitan environment in Lagos, Nigeria. 
III. The performance of the proposed model is evaluated by varying the network parameters for $900 \mathrm{MHz}$ and1800MHz bands.

The rest of this paper is organized as follows: Section II discusses the existing path loss prediction models. Section III describes the proposed model. Section IV presents the results and the discussions. Section V concludes this paper.

\section{SOME EXISTING PATH LOSS PREDICTION MODELS}

The average path loss in decibel $(\mathrm{dB})$ for an arbitrary transmitter and receiver considering the distance $d$ in an $m$ metropolitan terrain is given as:

$$
L_{m}(d)=L_{m}\left(d_{o}\right)+10 n \log \left(\frac{d}{d o}\right)
$$

where $d_{o}$ is the reference distance $L_{m}\left(d_{o}\right)$ is the average reference path loss in a metropolitan environment and $n$ is the path loss exponent, which indicates the rate at which the path loss increases with distance. The reference distance $d_{o}$ in a metropolitan environment must be selected such that it is in the far field of the transmitting antenna. The typical values mostly used are $1 \mathrm{~km}$ in microcell systems and $1 \mathrm{~m}$ in Pico cell systems ${ }^{[10]-[12]}$.

Equation (1) indicates the parameter of the path loss model. It is important to note that since the accuracy of the path loss model depends on these parameters to determine the value of $L_{m}\left(d_{o}\right)$, this may be predicted from the free space path loss model and the value of $n$ may be determined empirically.

\subsection{Hata Model}

The Hata Model was developed from the extensive empirical measurements taken by Okumura in Tokyo city, Japan. For suburban environments, the median path loss in $\mathrm{dB}$ is given by:

$$
L_{m}(d)=\alpha+\beta \log (d)
$$

Where

$$
\begin{gathered}
\alpha=69.55+26.16 \log \left(f_{c}\right)-13.82 \log \left(h_{b}\right)-a\left(h_{m}\right) \\
\beta=44.9-6.55 \log \left(h_{b}\right) \\
\gamma=5.4+2\left[\log \left(\frac{f c}{28}\right)\right]^{2}
\end{gathered}
$$

Also,

$$
a\left(h_{m}\right)=\left(1.1 \log \left(f_{c}\right)-0.7\right) h_{m}-\left(1.56 \log \left(f_{c}\right)-0.8\right)
$$

where $\alpha, \beta, h_{m}, a\left(h_{b}\right), f_{c}$ and $\gamma$ denote the correction factor that depends on frequency, correction factor for mobile antenna height, mobile antenna height, base station antenna height, frequency, and effective antenna gain respectively.

For suburban and non-urban models they are based on the urban model with the following corrections:

Path loss (sub-urban $)=$ path loss(urban $)-2\left[\log \left(\frac{f c}{28}\right)\right]^{2}-5.4$

And

Path $\operatorname{loss}($ non-urban $)=$ path $\operatorname{loss}($ urban $)-4.7[\log (f)]^{2}+18.33 \log (f)-40.94$

\subsection{Free Space Propagation model}

Path loss PL at a point $r$ is defined as the ratio of transmitted power at $r_{0}$ over the received power at $r, \operatorname{Pr}($ For free space propagation, the path loss is given as: 
$L(\mathrm{Db})=10 \log \frac{P t(r 0)}{d r(r)}=10 \log \left[\frac{G t G r 2}{d o}\right]$

where Gt and Gr are the gains of the transmitting antenna(Tx) and the receiving antenna (Rx) respectively. (Tx) and $(\mathrm{Rx})$ are separated by a distance $\mathrm{d}$ and is the wavelength in free space.

\section{PROPOSED MODEL}

In this paper, we propose a fuzzy-logic based path loss prediction model for metropolitan environments as illustrated in Fig. 1.

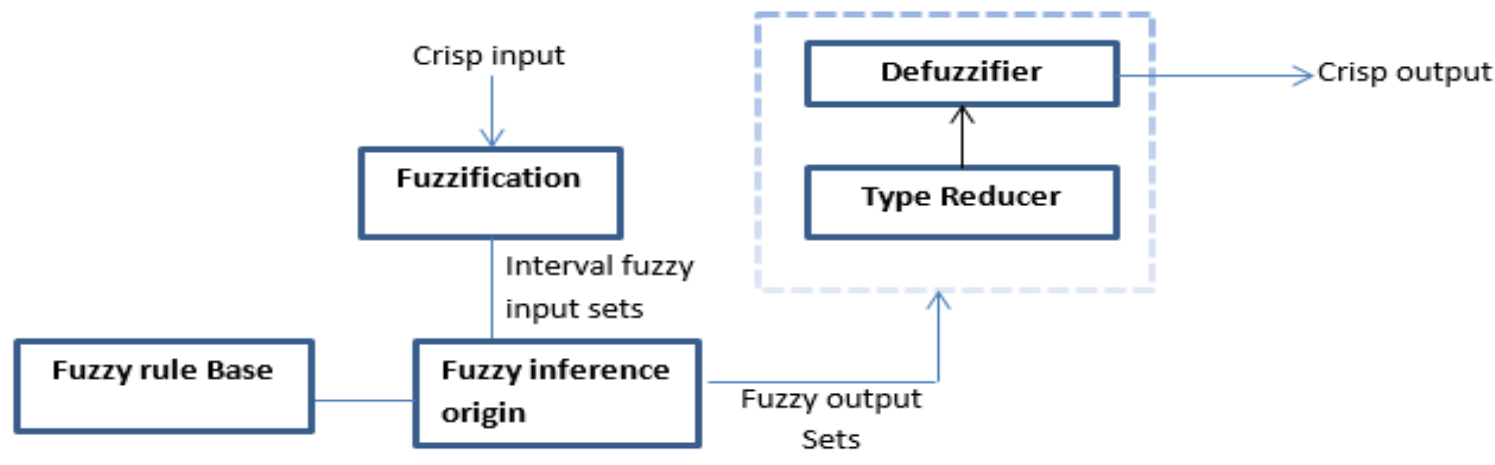

Figure 1. Block Diagram of a Generic Fuzzy Logic Type 2 Model

\subsection{Fuzzy logic}

Fuzzy logic applies fuzzy set theory in which a variable is a member of one or more sets with a specified degree of membership. In the literature, some researchers have also applied the fuzzy logic methods to compute nonlinear and ill-defined systems ${ }^{[13]-[14]}$. Though most of these methods focus on the type-1 fuzzy logic. Over the years, there has been an approach to model uncertainty which has called into play the type-2 fuzzy logic system. We have also observed that from a concept of the type-2 fuzzy logic, it is possible to predict the path loss using "IF-THEN" rules. The propagation environment inputs are converted by the fuzzifur to type-2 fuzzy set and later inference using the IF-THEN rule based on the path loss prediction outputs via the output processing. Moreover, it is worthy to note that the areas vary from terrain to terrain, requiring wide range databases, which is difficult to clutter terrain factors by the generic conventional method. This is the premise that led to the development of the type-2 fuzzy logic system for a metropolitan environment which yielded more accuracy in computation.

\subsection{Fuzzy Logic Type-2 system}

The arrangement of the type-2 fuzzy logic system is close to the structure of fuzzy logic system. The basic difference between them is the membership function and the output as shown in Fig. 1. For a type-1 Fuzzy logic system, the output processing block contains only the defuzzifier. The fuzzifier maps the crisp input into a fuzzy set, to become a type- 2 set. In the type-1 case, in the "IF THEN" rules, the $i$ th rule has the form "R": if $x 1=F 1$ " and $x 2$ = F2' and :::Xn is Fn'. Then, y is G', where x's are inputs; F1's are antecedent sets (i = 1:::p): y is the output; and G's are consequent sets. The common distinction between type 1 and type 2 is associated with the nature of the membership functions, which is not important while forming the rules, hence, the structure of the rules remain exactly the same as in the type 2 case. The proposed fuzzy-logic model for path loss prediction is shown in Fig. 2. 


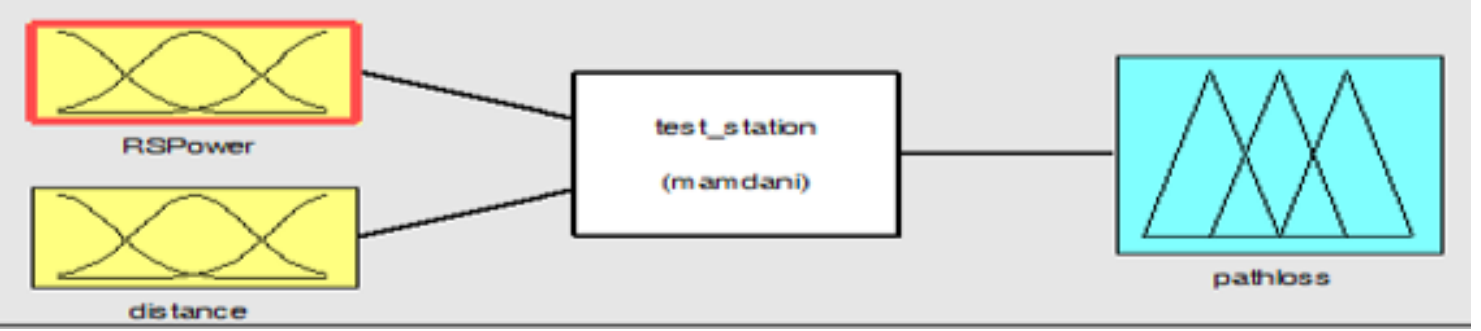

Figure 2. The Proposed Fuzzy-Logic Model for Path Loss Prediction.

However, it is noted that the only difference is that all of the sets involved are of type- 2 so the $i$ th rule in a type2 fuzzy logic system yield the form "R" "if $x_{1}, \hat{F}^{\prime}$ and $x_{2}$ is $\hat{F}^{\prime}$ and... $x_{p}$ is $\hat{F}^{\prime}$, THEN,yis $\bar{G}^{!}$.

\subsection{Fuzzy Inference}

The behavior of the system which relates the input and the output variables of the system is governed by a set of rules Atypical rule would be

$$
\begin{aligned}
& \text { If } x \text { is } A \text {, Then } y \text { is } B \\
& 150 \leq f_{c} \leq 220 M \mathrm{~Hz} \\
& 1 \leq h_{m} \leq 10 \mathrm{~m} \\
& 1 \leq d \leq 20 \mathrm{~km}
\end{aligned}
$$

If $x$ is $\mathrm{A}$, Then $y$ is $\mathrm{B}$, when a set if input variables are read, each of the rule that has any degree of truth in its premise is fixed and contributes to the form of the fuzzy-logic system (FLS) by approximately modifying it when all rules are fixed. The resulting system is expressed as a fuzzy set to represent the constraints output. The process is referred to as "Inference".

\subsection{Path loss Fuzzy Membership Function}

Fuzzy logic over the years has been used to model a lot of systems, which cannot be defined in an absolute manner. In this work, the RS-power and distance inputs have a Gaussian membership function type, while the path loss which is the output is of triangular membership function type.

\subsection{Defuzzification}

Defuzzification is the method used for conversion of fuzzy quantity into crisp quantity. There are many methods available for defuzzification. The most common one is the Centroid method, which utilizes the formula in equation ${ }^{(10)}$. In this paper, the Mamdani is chosen rather than the Sugeno ${ }^{[14]}$. The Mamdani FIS in this case is fed with two inputs namely the Received power (RSpower) and the distance of the user equipment (UE) while the output is the path loss of the channel. A 25-rules inference scheme is used for simplicity in order to avoid the complexity that comes with many rules if applied. Fig. 3 shows the defuzzification of the testing rule.

\section{RESULTS AND DISCUSSIONS}

Path loss exponents for seven environments were computed for fuzzy type 1 and 2 in $900 \mathrm{MHz}$ and $1800 \mathrm{MHz}$ and presented in Tables I-IV. The Ex-urban has the lowest path loss exponent of 5.11 in the $900 \mathrm{MHz}$ and 5.60 in the 1800MHzas can be seen from Tables I, III and II, IV respectively. This is due to the fact that signals are most likely not obstructed. The sub-urban area has the lowest prediction error. Line of sight can still be expected on some test locations. Although sites visited were located in cities where building heights, billboards and vegetation contribute to the increase in the path loss exponent from free space value of 2 . As seen from the Table V, the Mean Prediction error is less than $3.66 \mathrm{~dB}$. Furthermore, path loss exponents are observed to become smaller as the received signal strength (RSS) increases and at shorter distances. 
RSPower $=10$

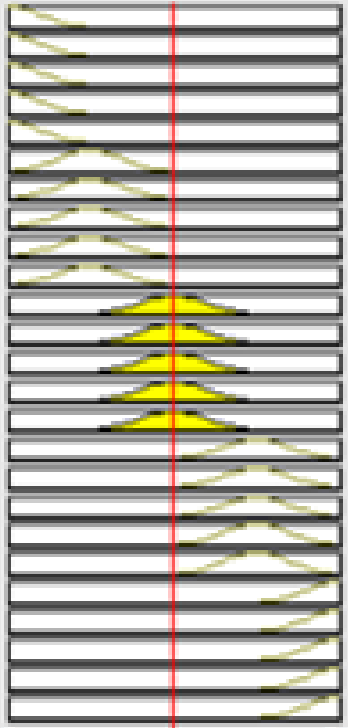

distance -10

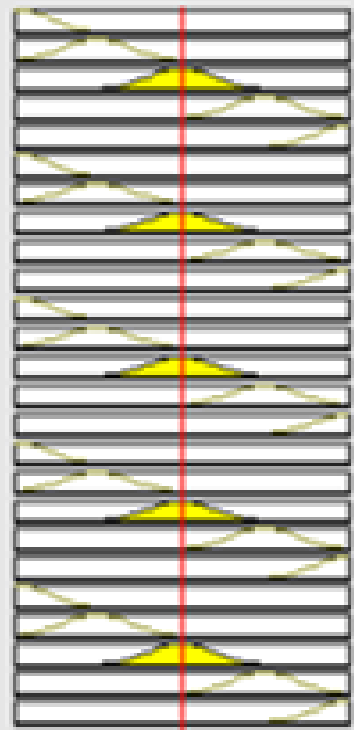

pathibss $=90$

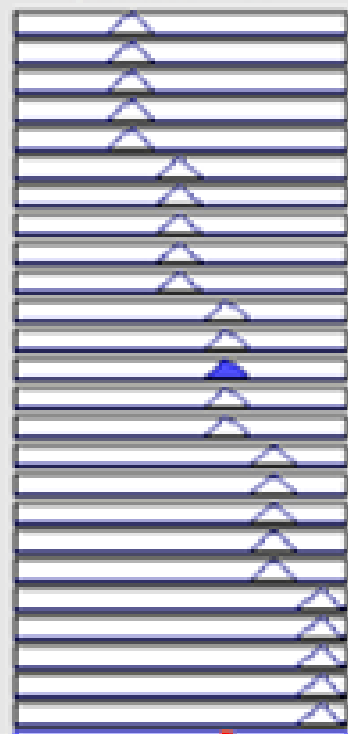

Figure 3. Defuzzification of the Testing Rule.

Table I .900MHZ -FUZZY TYPE I

\begin{tabular}{|l|l|l|}
\hline Environment & Path loss Exponent & Relative Error \\
\hline Non Urban & 6.768421053 & $7.88741 \mathrm{E}-08$ \\
\hline Urban & 5.269172932 & $-7.68201 \mathrm{E}-07$ \\
\hline Sub urban & 6.180451128 & $1.42554 \mathrm{E}-15$ \\
\hline Ex Urban & 5.105263158 & $4.09192 \mathrm{E}-06$ \\
\hline Dense Urban & 7.051128 & $5.51365 \mathrm{E}-07$ \\
\hline Micro Urban & 6.196992481 & $-8.01716 \mathrm{E}-07$ \\
\hline Peri Urban & 7.13283208 & 0.03783157 \\
\hline
\end{tabular}

Table II.1800MHZ -FUZZY TYPE 1

\begin{tabular}{|l|l|l|}
\hline Environment & Path loss Exponent & Relative Error \\
\hline Non Urban & 5.780451128 & 0.035545629 \\
\hline Urban & 5.936842105 & 0.068674113 \\
\hline Sub urban & 5.869172932 & 0.113569189 \\
\hline Dx Urban & 5.598496241 & 0.129029705 \\
\hline Micro Urban & 5.866165414 & 0.157775717 \\
\hline Peri Urban & 7.132330827 & -0.112865623 \\
\hline
\end{tabular}

Table III.900MHZ -FUZZY TYPE 2

\begin{tabular}{|l|l|l|}
\hline Environment & Path loss Exponent & Relative Error \\
\hline Non Urban & 6.768421053 & $2.38184 \mathrm{E}-07$ \\
\hline Urban & 5.269172932 & $-1.10724 \mathrm{E}-05$ \\
\hline Sub urban & 6.180451128 & $-2.25122 \mathrm{E}-14$ \\
\hline Ex Urban & 5.105263158 & $9.70393 \mathrm{E}-06$ \\
\hline Dense Urban & 7.051128 & $1.32682 \mathrm{E}-06$ \\
\hline Micro Urban & 6.196992481 & $-2.50423 \mathrm{E}-06$ \\
\hline Peri Urban & 7.13283208 & 0.1910968 \\
\hline
\end{tabular}

Measured path loss values were compared to fuzzy path loss models and they are in good agreement with Okumura Hata models. Its regression is evident in accordance to distance and antenna heights. The value of 
constant offset is at the highest in Urban environments being $60 \mathrm{~dB}$ for $900 \mathrm{MHz}$ but the Ex-urban environment of the $1800 \mathrm{MHz}$ has a constant offset of

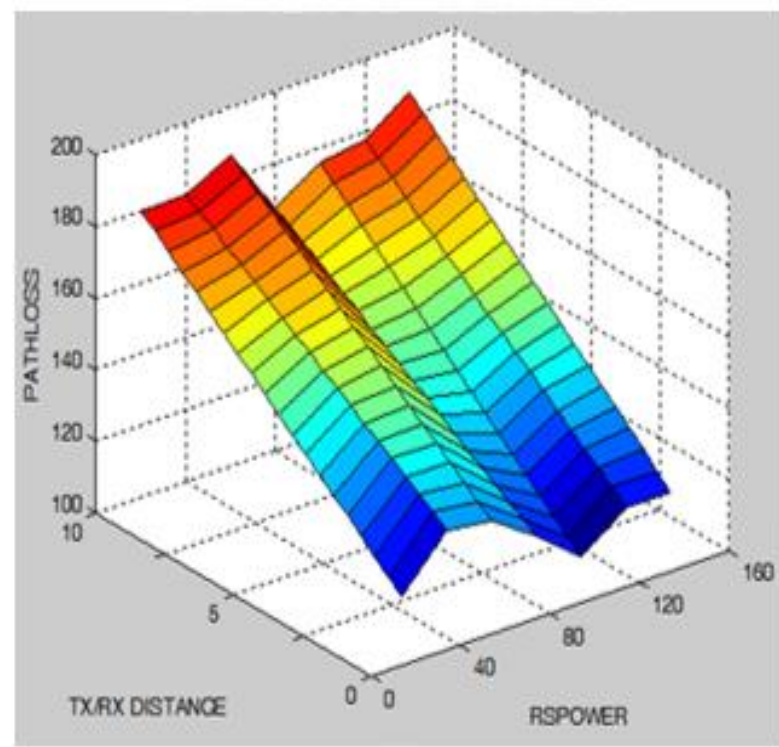

Case I: Path loss from Linear Regression Model for $900 \mathrm{MHz}$

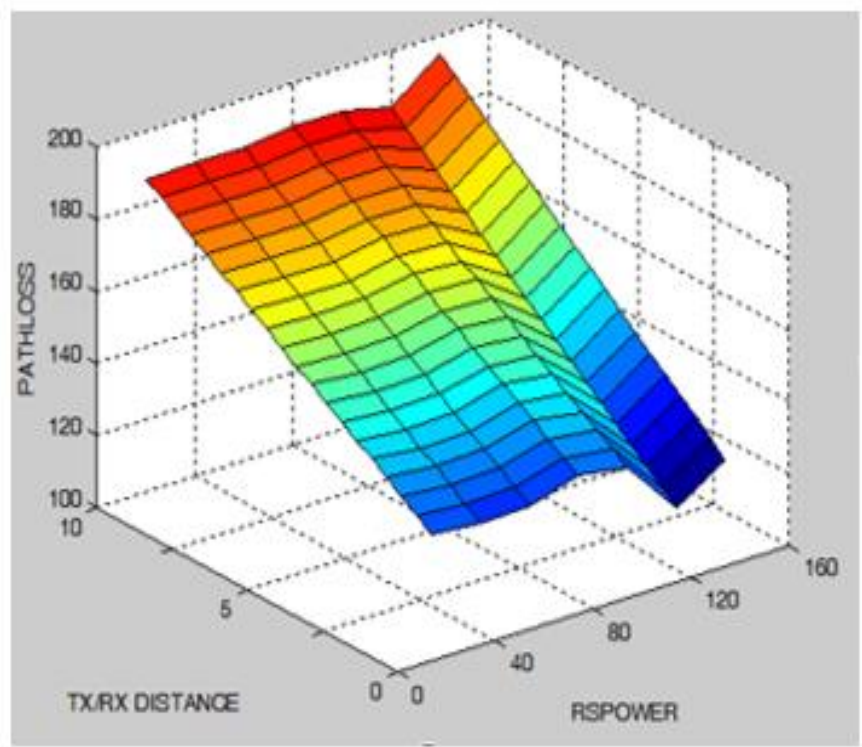

Case II: Path loss from Linear Regression Model for $1800 \mathrm{MHz}$

Figure 4. Comparison of $900 \mathrm{MHz}$ and $1800 \mathrm{MHz}$ of Linear Regression model.
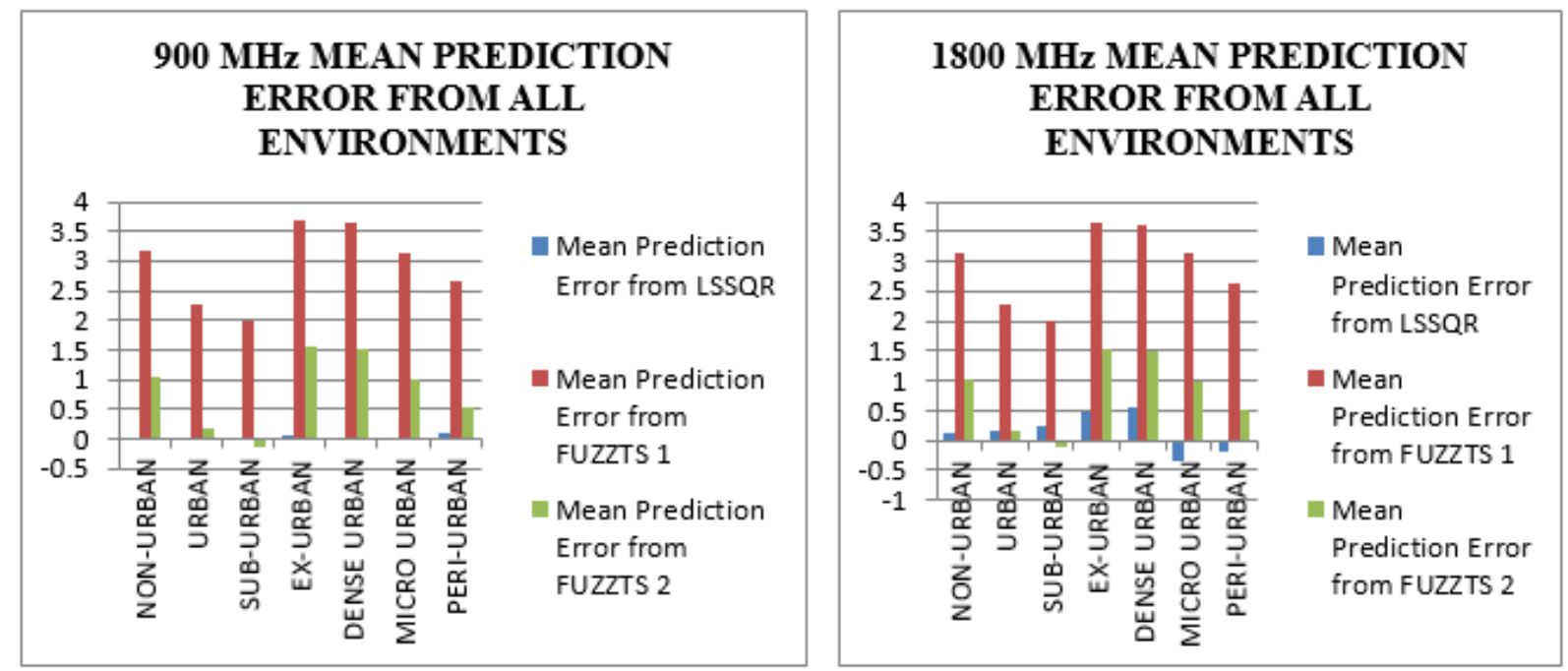

Figure 5. Comparison of Mean Prediction Error from Least Square Regression (LSSQR), Fuzzy type 1 (FUZZTS 1) and Fuzzy type 2 (FUZZTS 2)

$64 \mathrm{~dB}$. This occurs due to the obstruction and the line-of sight connection is lost earlier than assumed comparing with the assumed reference point. Moreover, propagation behind the corners of buildings was observed to cause 20-25 Db attenuation to the path loss values in urban environment. Fig4 demonstrates the comparison for linear regression model for $900 \mathrm{MHz}$ and $1800 \mathrm{MHz}$. It is observed that higher path loss values are obtained at 1800 $\mathrm{MHz}$ than at $900 \mathrm{MHz}$. This is due to early connection loss. The mean prediction error gives a summary of the analyzed data in the units of the dependent variable as shown in Fig. 5. The proposed model gives mean 
prediction error of 1.6dB as compared to LSSQR and FUZZTS1. Fig. 6 shows the comparison of the proposed model for $900 \mathrm{MHz}$ and $1800 \mathrm{MHz}$. Almost the same path loss values were obtained, this shows that the proposed model is a reliable tool in estimating outdoor power levels. Hence, the fuzzy-logic based model can be applied to both range of signal strength at $900 \mathrm{MHz}$ and $1800 \mathrm{MHz}$ for better path loss prediction.

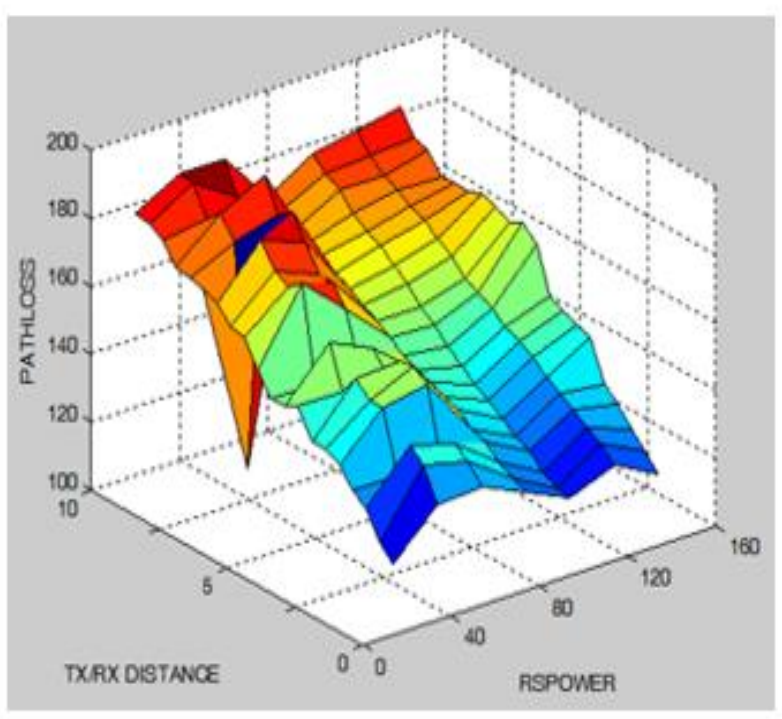

Case I: Path loss from fuzzy type-2 model for $900 \mathrm{MHz}$

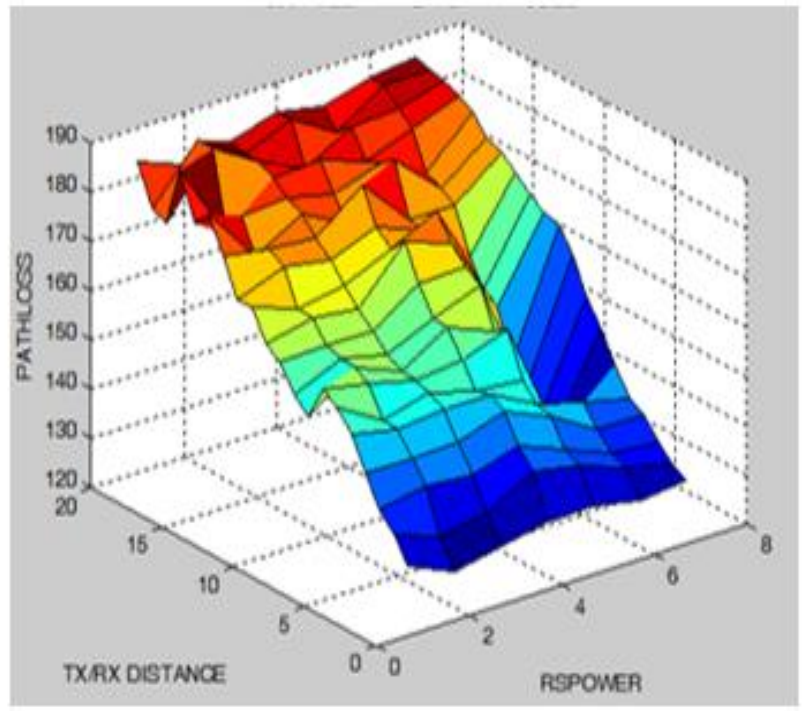

Case II: Path loss from fuzzy type-2 model for $1800 \mathrm{MHz}$

Figure 6. Comparison of $900 \mathrm{MHz}$ and $1800 \mathrm{MHz}$ of fuzzy type-2 model.

Table IV.1800MHZ -FUZZY TYPE 2

\begin{tabular}{|l|l|l|}
\hline Environment & Path loss Exponent & Relative Error \\
\hline Non Urban & 6.768421053 & 0.10836075 \\
\hline Urban & 5.269172932 & 0.991204417 \\
\hline Sub urban & 6.180451128 & -1.833281284 \\
\hline Ex Urban & 5.105263158 & 0.306100803 \\
\hline Dense Urban & 7.051128 & 0.380499613 \\
\hline Micro Urban & 6.196992481 & -0.352129976 \\
\hline Peri Urban & 7.13283208 & -0.352488794 \\
\hline
\end{tabular}

Table V. 1800MHZ -FUZZY TYPE 2

\begin{tabular}{|l|l|l|c|l|l|}
\hline & & \multicolumn{3}{|l|}{900 MHz Station } & \multicolumn{1}{l|}{ 1800 MHz Station } \\
\hline S/N & Environment & $R^{2}$ & RMSE & $R^{2}$ & RMSE \\
\hline 1 & Non Urban & 0.98 & 2.44 & 0.91 & 5.52 \\
\hline 2 & Urban & 0.45 & 16.71 & 0.93 & 4.55 \\
\hline 3 & Sub urban & 0.87 & 6.84 & 0.97 & 3.05 \\
\hline 4 & Ex Urban & 0.99 & 0.52 & 0.93 & 4.41 \\
\hline 5 & Dense Urban & 0.99 & 1.36 & 0.94 & 4.38 \\
\hline 6 & Micro Urban & 0.99 & 0.77 & 0.90 & 6.91 \\
\hline 7 & Peri Urban & 0.99 & 6.29 & 1.07 & 3.99 \\
\hline
\end{tabular}

\section{CONCLUSION}

In this paper, we have proposed a fuzzy logic based path loss prediction model for metropolitan environment. The performance of the proposed model has been evaluated at $900 \mathrm{MHzand} 1800 \mathrm{MHz}$ bands. The proposed model is more efficient, faster and accurate than the physical and empirical methods. In addition, it can be used 
to cover large distances and it has a potential in telecommunication industry for link budget and in the determination of the coverage area of a base station.

\section{REFERENCES}

[1] Rappaport, T. S., "Wireless Communications: Principles and Practice"2nd Edition, Prentice Hall, Upper Saddle River, New Jersey, USA, 2002.

[2] S. Faruque, "Propagation Prediction Based on Environmental Classification and Fuzzy Logic Approximation,” in Proc. IEEE ICC, pp. 272-276,Jun. 1996.

[3] P. Phetsri, A. Sungkhapong and S. Phaiboon, "Micro Cell Path Loss Prediction through Hilly Forest Terrain: A Case Study in South of Thailand,” in Proc. AIMSEC, pp. 4670-4673, Aug. 2011.

[4] A. Obot, O. Simeon, and J. Afolayan "Comparative Analysis of Path Loss Prediction Models for Urban Macrocellular Environment," Nigerian Jour. of Tech., vol. 30, no. 3, pp. 50-59, Oct. 2011.

[5] J. Chebil, A. Lawas and M. Islam, "Comparison Between Measured and Predicted Path Loss for Mobile Communication in Malaysia," World Appl. Sciences Jour. 21 (Math. Appl. in Eng.,) pp. 123-128, Oct. 2013.

[6] S. Mathew, K. Shylaja, T. Jayasri and M. Hemalatha, "Path Loss Prediction in Wireless Communication System using Fuzzy Logic,” Indian Jour. of Sci. and Tech., vol. 7, no. 5, pp. 642-647, May. 2014.

[7] J. Choi, H. Oh, H. Jeon, "Propagation Prediction for LTE Small Cells with Antenna Beam Tilt," IEEE VTC pp. 1-5, Sept. 2014.

[8] J. Eichie, O. Oyedum, M. Ajewole, and A. Aibinu, "Comparative Analysis of Basic Models and Artificial Neural Network Based Model forPath Loss Prediction,” Progress In Electromagnetics Research Vol. 61,pp. 133-146, Aug. 2017.

[9] Nwalozie G. C., S. U. Ufoaroh, C. O. Ezeagwu, and A. C. Ejiofor, "Path loss prediction for GSM mobile networks for urban region of Aba, South-East, Nigeria," International Journal of Computer Science and Mobile Computing, Vol. 3, No. 2, pp. 267281, 2014.

[10] Ogbulezie, J. C., M. U. Onuu, J. O. Ushie, and B. E. Usibe, "Propagation models for GSM 900 and 1800 MHz for Port Harcourt and Enugu, Nigeria,” Netw. and Commun. Techn., Vol. 2, No. 2, pp. 110, 2013.

[11] Benmus, T. A., R. Abboud, and M. K. Shater, "Neural network approach to model the propagation path loss for great Tripoli area at 900, 1800 and2100 MHz bands," International Journal of Sciences and Techniques of Automatic Control and Engineering, Vol. 10, No. 2, pp. 21212126, 2016.

[12] Q. Liang and J.M. Mendil Interval Type-2 Fuzzy Logic Systems: Theory and Design IEEE Trans Fuzzy Set. Vol. 8 no 5 pp. 535-550, oct. 2000.

[13] L. Dutu, G. Mauris and P. Bolon "A fast and accurate rule based generation method for mamdani fuzzy systems,” IEEE Trans. on Fuzzy Sys., vol. 26, no. 2, 715733, 2018.

[14] O. Shoewu and L.I. Oborkhale "Path Loss Modelling of Less Dense Urban Area in Lagos State Using Lee Model" Journal of Advancement in Engineering and Technology, Vol. 6, No. 7, pg. 8 - 16, 2017. 